\title{
PRELIMINARY DEVELOPMENTS OF A TWO-FLUID CARTESIAN-GRID EXPLICIT FINITE-VOLUME MODEL FOR MARINE APPLICATIONS.
}

\author{
C. Leroy, P. Bigay, G. Oger, D. Le Touzé and B. Alessandrini
}

LUNAM Université, Ecole Centrale Nantes, LHEEA Lab. (UMR CNRS), Nantes, France

(clement.leroy@ec-nantes.fr)

\begin{abstract}
An explicit Finite Volume method for solving hydrodynamic flows is presented in this paper. These developments are based on an explicit cell-centered scheme solving the compressible fluid equations in a pseudo-compressible strategy where second-order accuracy is provided by using a MUSCL scheme together with various limiters for the hyperbolic part. In this recent model, boundaries are handled through a Cut-Cell method, so that solids as well as fluid interfaces are explicitly moved in a non-diffusive way, ensuring local mass conservation within fluids. An improved cut-cell algorithm based on the Voxel traversal algorithm coupled with a local Floodfill Scanline has been developed, in order to handle boundaries of arbitrary geometrical complexity. To cope with small cells instability problems near the boundaries, a fully conservative merging method is implemented. In this paper, this solver is validated on 2-D hydrodynamic test cases, such as flows past obstacles. Test cases involving large body movement are then performed and discussed. The latter test cases are performed both in the frame of the body and in a fixed frame where the body is moving across the grid. Then, a two-fluid formulation is introduced in the model and described in detail in the present paper. First validations of this two-fluid formulation are eventually presented.
\end{abstract}

Keywords: Compressible Solver, Two-fluid model, Cartesian grid, Embedded boundary.

\section{INTRODUCTION}

Numerical simulation is more and more widely used in hydrodynamics. Limited to simplified models of the flow around a body in frequency domain two decades ago, it now deals with the temporal modeling of complex unsteady phenomena. In particular, models based on the Navier-Stokes equations in Reynolds averaging (RANS) are now used routinely in applied research to solve complex realistic problems. Meanwhile, the commercialization of general codes of this kind knows an increasing success in the industry, starting to replace the previous empirical solutions. The RANS models based on volume mesh implicit methods still do not respond to all situations encountered in naval hydrodynamics and offshore. One can give as examples the simulation of two-phase phenomena caused by the progression of a ship at sea with its propeller (air entrainment in the jet bow, bubbles in the wake), violent sloshing impacts on long duration, etc. These examples raise the problem of safety of floating structures, their staff, their passengers and their cargo (often polluting) but they also concern energy reduction, ship signature, etc. The main limitations faced by standard solvers based on implicit methods on body-fitted unstructured meshes are: the presence of multiple bodies of 
complex geometry in arbitrary motion in the flow, non-diffusive interfaces between several fluids, multi-physics within the solver, automatic mesh refinement, mesh adaptation in the frame of fluid-structure coupling. Here, we develop a different model based on a fixed Cartesian grid, an explicit resolution based on compressible Finite Volumes enabling easy inclusion of multi-physics. Arbitrary complex geometries can be embedded in the fixed grid and move freely thanks to a cut-cell technique. Fully-conservative treatment of interfaces is presently being implemented. In the present paper, these different components are first presented, and followed by validation test cases.

\section{EXPLICIT FINITE VOLUME SOLUTION FRAMEWORK}

\subsection{Navier-Stokes \& Inviscid Euler Equations}

We model 3-D Navier-Stokes equations for viscous compressible flow:

$\overrightarrow{W_{t}}+F \overrightarrow{(W)_{x}}+G \overrightarrow{(W)_{y}}+H \overrightarrow{(W)_{z}}=\overrightarrow{V u s c}$

which can be written in conservative form as

$$
\left(\begin{array}{l}
\rho \\
\rho u \\
\rho v \\
\rho w \\
\rho E
\end{array}\right)_{t}+\left(\begin{array}{l}
\rho u \\
\rho u^{2}+P \\
\rho u v \\
\rho u w \\
(\rho E+P) u
\end{array}\right)_{x}+\left(\begin{array}{l}
\rho v \\
\rho v u \\
\rho v^{2}+P \\
\rho v w \\
(\rho E+P) v
\end{array}\right)_{y}+\left(\begin{array}{l}
\rho w \\
\rho w u \\
\rho w v \\
\rho w^{2}+P \\
(\rho E+P) w
\end{array}\right)_{z}=\overrightarrow{V i s c}
$$

The internal energy is linked to the total energy per unit volume and the kinetic energy by

$$
E_{\text {tot }}=\rho E+\frac{\rho\left(u^{2}+v^{2}+\right.}{2}
$$

An additional equation is needed to close the system by coupling the pressure with conservative variables $\operatorname{EOS}(\rho, \rho \mathrm{U}, \rho \mathrm{E})=0$. We use either an ideal gas assumption or a barotropic fluid. On the one hand Tammann-Gibson's equation of state expresses the pressure $P$ as a function of the density $\rho$, the internal energy $E$ and the polytrophic constant $\gamma$.

$$
P=(\gamma-1) \rho E=(\gamma-1)\left(E_{\text {tot }}-\frac{\rho\left(u^{2}+v^{2}+w^{2}\right)}{2}\right)
$$

On the other hand Tait's equation writes the pressure as a function of the density, the polytrophic constant $\gamma$, a reference pressure $P_{0}$, the nominal density $\rho_{0}$ and the nominal speed of sound $c_{0}$

$$
P-P_{0}=\frac{\rho_{0} c_{0}^{2}}{\gamma}\left[\left(\frac{\rho}{\rho_{0}}\right)^{Y}-1\right]
$$

The viscosity terms in Navier-Stokes equation can be represented in terms of the viscous stress tensor components as : 


$$
\overrightarrow{V u s c}=\left(\begin{array}{c}
\left(\begin{array}{c}
\tau_{x x} \\
\tau_{x y} \\
\tau_{x z}
\end{array}\right)_{x}+\left(\begin{array}{c}
\tau_{x y} \\
\tau_{y y} \\
\tau_{y z}
\end{array}\right)_{y}+\left(\begin{array}{l}
\tau_{x z} \\
\tau_{y z} \\
\tau_{z z}
\end{array}\right)_{z} \\
\vec{\nabla}\left(\begin{array}{l}
u \tau_{x x}+v \tau_{x y}+w \tau_{x z} \\
u \tau_{x y}+v \tau_{y y}+w \tau_{y z} \\
u \tau_{x z}+v \tau_{y z}+w \tau_{z z}
\end{array}\right)
\end{array}\right)
$$

\section{FINITE VOLUME CHARACTERISTIC FLUX (FVCF) SCHEMES}

The FVCF scheme, proposed by Ghidaglia et al [1] in 1996, is a finite volume scheme with cell centered variables, including the kinetic vector. The conservation laws are discretized by calculating numerical sum fluxes $\phi_{F V C F}$ through cell edges. These fluxes are expressed in terms of cell defined physical fluxes $F, G$ and $H$ in 3-D, and not cell variables $W$ as classical Godunov scheme. Introducing the flux of conservative variables in normal direction $\vec{n}$ as

$$
\Psi(W, \vec{n})=\vec{W}(\vec{U} \cdot \vec{n})+P\left(\begin{array}{c}
0 \\
\vec{n} \\
(U \cdot \vec{n})
\end{array}\right)
$$

Let us define $V(t)$ a volume and its boundary $\delta V(t)$ which can be decomposed in time independent edges $\Gamma$ and moving edges $\Xi(\mathrm{t})$ with local velocity $\vec{U}_{\mathrm{int}}$.

Introducing (7) one gets

$$
\iint_{g(\mathrm{t})} \Psi(W, \vec{n}) d S=\iint_{g(\mathrm{t})} \vec{W}\left(\vec{U}_{\text {int }} \cdot \vec{n}\right) d S+\iint_{\mathrm{g}(\mathrm{t})} P_{\text {int }}\left(\begin{array}{c}
0 \\
\vec{n} \\
\left(\vec{U}_{\text {int }} \cdot \vec{n}\right)
\end{array}\right) d S
$$

The integrated system becomes:

$$
\frac{d}{d t} \iiint_{V(t)} \vec{W} d \vartheta+\iint_{\Xi(t)} P_{\text {int }}\left(\begin{array}{c}
0 \\
\vec{n} \\
\left(\vec{U}_{\text {int }}, \vec{n}\right)
\end{array}\right) d S+\iint_{\Gamma} \Psi(W, \vec{n}) d S=0
$$

In the finite volume framework we obtain:

$$
\frac{V o l^{n+1} \cdot \overrightarrow{W^{n+1}}-V o l^{n} \cdot \overrightarrow{W^{n}}}{d t}+A \cdot P_{\text {int }}\left(\begin{array}{c}
0 \\
\vec{n} \\
\left(\vec{U}_{\text {int }} \cdot \vec{n}\right)
\end{array}\right)+A \cdot \emptyset_{\text {FVCF }}=0
$$

where $d t$ is the timestep, superscripts $n$ and $n+1$ indicate the time levels, $\vec{n}$ is the unit normal vector to the face, and $A$ the face area. We discretize the Navier-Stokes equation as a hyperbolic part plus some viscosity terms. We use the Jacobian matrix $\overline{\bar{J}}$ associated to the hyperbolic system and the Tait's equation of state to build a flux solution $\phi_{F V C F}$. For this study we can rewrite (2): 


$$
W_{t}+\sum_{i=1}^{\operatorname{dim}} \overline{\bar{J}}\left(W, \overrightarrow{e_{i}}\right) F(W)_{x_{i}}=0
$$

We then need to multiply (11) by the same Jacobian matrix expressed at the mid interface of the two cells. MUSCL method can be used here to improve the reconstruction of the new interface conservative variables that will be explained later with more details. We use simple linear interpolation as follows, where $L$ and $R$ refer respectively to the left and right states of the mid interface.

$$
\left\{\begin{array}{l}
(F(W) \cdot \vec{n})_{t}+\bar{J}\left(W_{\mathrm{int}}, \vec{n}\right) \cdot(F(W) \cdot \vec{n})_{n}=0 \\
W_{\mathrm{int}}=\frac{\operatorname{Vol}_{L} W_{L}+\operatorname{Vol}_{R} W_{R}}{\operatorname{Vol}_{L}+\operatorname{Vol}_{R}}
\end{array}\right.
$$

For barotropic fluids, hyperbolic conservation law Jacobian's is:

$$
\overline{\bar{l}}=\frac{\partial \Psi(W, \vec{n})}{\partial W}=\left(\begin{array}{cc}
0 & \vec{n} \\
c^{2} \vec{n}-(\vec{u} \cdot \vec{n}) \vec{u} & \vec{u} \otimes \vec{n}+\vec{u} \cdot \vec{n} I d_{\operatorname{dim}+2}
\end{array}\right)
$$

With energy conservation equation Jacobian's matrix becomes:

$$
\overline{\bar{l}}=\left(\begin{array}{ccc}
0 & \vec{n} & 0 \\
\Theta \vec{n}-(\vec{u} \cdot \vec{n}) \vec{u} & \vec{u} \otimes \vec{n}-\kappa \vec{n} \otimes \vec{u}+\vec{u} \cdot \vec{n} I d_{d i m+2} & \kappa \vec{n} \\
(\Theta-H)(\vec{u} \cdot \vec{n}) & H \vec{n}-\kappa(\vec{u} \cdot \vec{n}) \vec{u} & (1+\kappa)(\vec{u} \cdot \vec{n})
\end{array}\right)
$$

The next step consists in computing eigensystem see in [2] for more details.

We recall here two classical forms of calculation fluxes:

The Godunov flux [15] reads:

$$
\phi_{\text {Godunov }}=\psi\left(W_{e}\left[W_{l}, W_{r}\right], \vec{n}\right)
$$

where $W_{e}\left[W_{l}, W_{r}\right]$ is the exact solution of a Riemann problem between left and right states.

The Roe flux [16] reads:

$$
\emptyset_{R O E}=\frac{\Psi\left(W_{r}, \vec{n}\right)+\Psi\left(W_{1}, \vec{n}\right)}{2}-\left|A_{R O E}\right| \frac{W_{r}-W_{0}}{2}
$$

The algebraic reduction provides us with everything to work in the characteristic basis. The FVCF flux $\phi_{F V C F}$ can then be written in physical space in the following form 


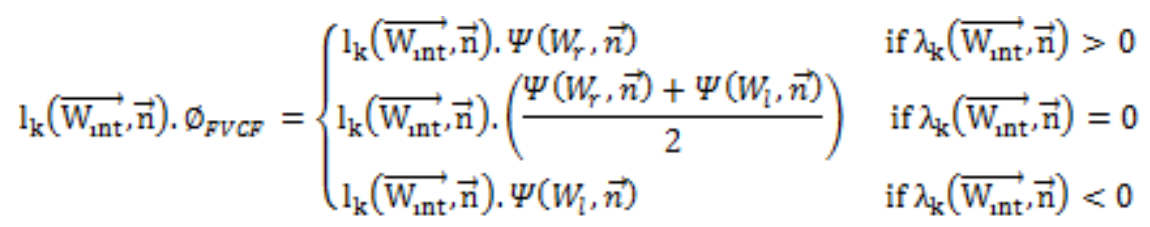

That can be sum up in general upwind equation

$$
\emptyset_{\text {FVCF }}=\frac{\Psi\left(W_{\gamma}, \vec{n}\right)+\Psi\left(W_{1}, \vec{n}\right)}{2}-\overline{\bar{R}} \cdot \operatorname{Matrix}_{\text {sign }(\bar{D})} \cdot \overline{\bar{L}} * \frac{\Psi\left(W_{\gamma}, \vec{n}\right)-\Psi\left(W_{1}, \vec{n}\right)}{2}
$$

Remark 1. FVCF method has a close definition with the Roe method, nonetheless the Roe matrix $A_{R O E}$ is not always calculable in complex cases as multiphase flows unlike Matrix ${ }_{\operatorname{sign}(\overline{\bar{D}})}$. Remark 2. FVCF method remains consistent in any linearization case of the left and right states.

Remark 3. FVCF method naturally translates interface exchange between adjacent cells.

The timestep is defined by the following CFL condition:

$$
d t \leq \min _{i}\left(\frac{\operatorname{Vol}_{i}}{\Gamma_{\mathrm{int}} \max _{k}\left|\left(\lambda_{i}\right)_{k}\right|}\right)
$$

where $\Gamma_{\text {int }}$ is the area of the interface between two adjacent cells.

\section{CHARACTERISTIC BOUNDARY CONDITIONS}

Inlet-Outlet boundary conditions are written into the characteristic space, see [1]. Inflow velocity is imposed at the inlet boundary, which allows calculating density and then pressure through the EOS.

$$
\left\{\begin{array}{c}
l_{\text {DIM }}\left(\overrightarrow{W_{\text {Int }},} \vec{n}\right) \cdot \overrightarrow{W_{\text {Inflow }}}=l_{\text {DIM }}\left(\overrightarrow{W_{\text {Int }}}, \vec{n}\right) \cdot \vec{W} \\
\Rightarrow>\rho_{\text {inflow }}=\frac{\rho c}{c+\left(\overrightarrow{U_{\text {Inflow }}}-\vec{U}\right) \vec{n}}
\end{array}\right.
$$

The outlet boundary condition relies in the imposition of the outflow pressure from which we calculate the density.

$$
P_{\text {out flow }}=>\rho_{\text {outflow }}=\rho\left(\frac{\gamma \cdot P_{\text {outflow }}}{\rho_{0} c_{0}^{2}}+1\right)^{\frac{1}{Y}}
$$


At a wall, where reflecting boundary conditions should be imposed, we consider the normal velocity to be equal to zero. As a result, the numerical flux at a wall is simply defined using the characteristic the pressure as

$$
\left\{\begin{array}{c}
\emptyset_{\text {FVCF }}^{\text {Wall }}=P_{\text {waIl }}\left(\begin{array}{l}
0 \\
\vec{n}
\end{array}\right) \\
l_{\text {DIM }}(\vec{W}, \vec{n}) \cdot P_{\text {wall }}\left(\begin{array}{l}
0 \\
\vec{n}
\end{array}\right)=l_{\text {DIM }}(\vec{W}, \vec{n}) \cdot \Psi(\vec{W}, \vec{n}) \\
\Rightarrow>P_{\text {wall }}=P+\rho c(\vec{U} \cdot \vec{n})
\end{array}\right.
$$

\subsection{Characteristic hybrid cut-cell method}

Flow simulation around moving complex bodies is a challenging problem, especially when a fixed Cartesian grid is used. We have to find local grid modification on body surface, without significant increasing computational cost. We present here a new implementation of an existing two-dimensional cell-merging method to overcome the problem of conservation laws. The present method may have a better potential for 3-D extension. The first step relies in immersing a 2-D or 3-D complex geometry in a Cartesian grid and to provide volumes, intersection coordinates and solid edge normals for each intersected cell. Instead of using a polygon clipping algorithm [3] we use the Voxel traversal algorithm [10] coupled with a local floodfill scanline to intersect 2-D or 3-D complex surface meshes with fix Cartesian grids. The second step consists in defining the cut-cell topologies which can be found in 2-D/3-D [7], and to define a modified FVCF scheme. Considering the 2-D problem for simplicity, 12 configurations exist which can be viewed as 6 symmetric configurations [2]. These cell configurations modify the discretized scheme since 3 to 5 flux calculations should now be performed for each cell on specific non-equal edges. The finite volume scheme becomes:

$$
W_{i, j}^{n+1}=W_{i, j}^{n}-\frac{d t}{V o l_{i, j}} \sum_{k \in \in d g e s} \delta l^{(k)} \cdot \varphi_{F V C F}^{G)}
$$

where $d t$ is a timestep, superscripts $n$ and $n+1$ indicate the time levels, edges is the total number of cell faces in the cell under consideration, $k$ is the face number, and $\delta l^{\mathrm{k})}$ is the face area.

(1) Computing the total flux of all cells at time level $n$;

(2) Merging the cut cells;

(3) Moving the body to the next time level, $n+1$;

(4) Computing cell geometrical properties;

(5) Computing the solution;

(6) Separating the merged cut cells.

Cell merging treats very small cut cells, which can greatly reduce the size of a timestep. Furthermore, it has been shown by Coirier et al.[17] that cell merging affects only the local computational accuracy, not its global value. However Cut-Cell configurations induce the presence of small volume cells leading to low local timesteps imposed by the CFL criterion. A fully conservative cell-merge method is thus added to avoid this difficulty. We thus define 
a minimum volume under which a small cell is merged with it closest neighbor cell. This technique results in a lower local accuracy but has no significant effect on global accuracy, see [11]. Since van Leer's work [4], the MUSCL scheme (Monotonic Upstream-Centered Scheme for Conservation Laws) has been studied and widely used in compressible fluid simulations. In MUSCL schemes, the conservative solution vectors are described as piecewise linear polynomials for recovering second-order accuracy. A gradient ratio is introduced in each direction. We write a new conservative vector at the edge between two conform Cartesian cells using the two closest neighbor cells. A monotonic slope limiter is used to forbid non realistic or inversion reconstruction. The Minmod slope limiter function preserves a strict monotonic reconstruction but in practice we use the Monotonized Central (MC) limiter, which we consider it provides better results.

\subsection{Validations}

As a first validation test case, we study an inviscid flow past a fixed cylinder, with an imposed incident velocity of $1 \mathrm{~m} / \mathrm{s}$ (Figure 3). This cylinder is located in the center of a 20 meter long infinite tank. As a second step of this study, the solution obtained on this fixed cylinder is then compared to the case of a cylinder moving with an imposed velocity of $1 \mathrm{~m} / \mathrm{s}$ in a zero velocity flow field. Such conditions are equivalent, so that identical solutions can be expected. In both cases we use potential flow result as an analytical solution.
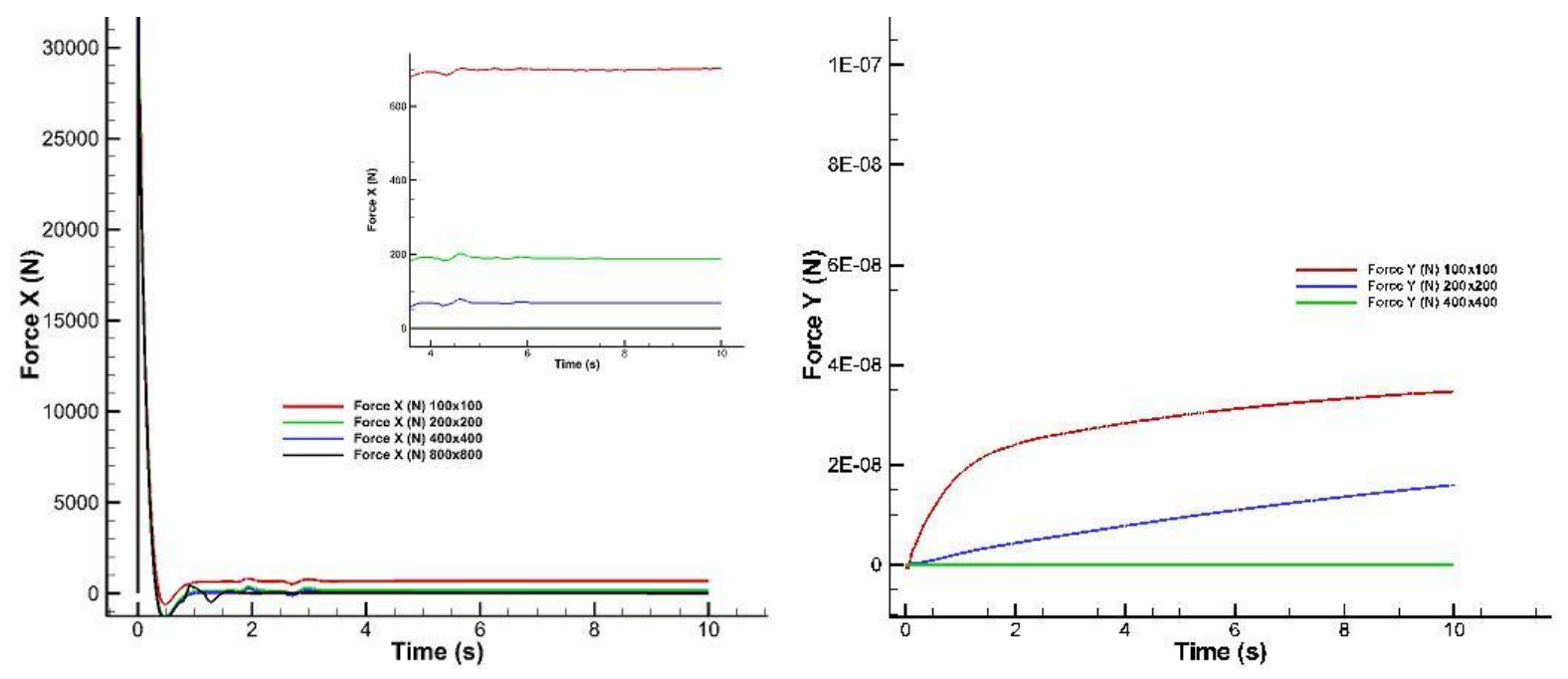

Figure 1. Force $\mathrm{X}$ and $\mathrm{Y}$ history on the fixed cylinder.

After a convergence study, all present results converge through 0 on $\mathrm{X}$ and $\mathrm{Y}$ direction, in respect of inviscid flow hypothesis. We now compare the angular local pressure on the cylinder once the steady flow reached with a generate potential reference solution (Figure 2). 


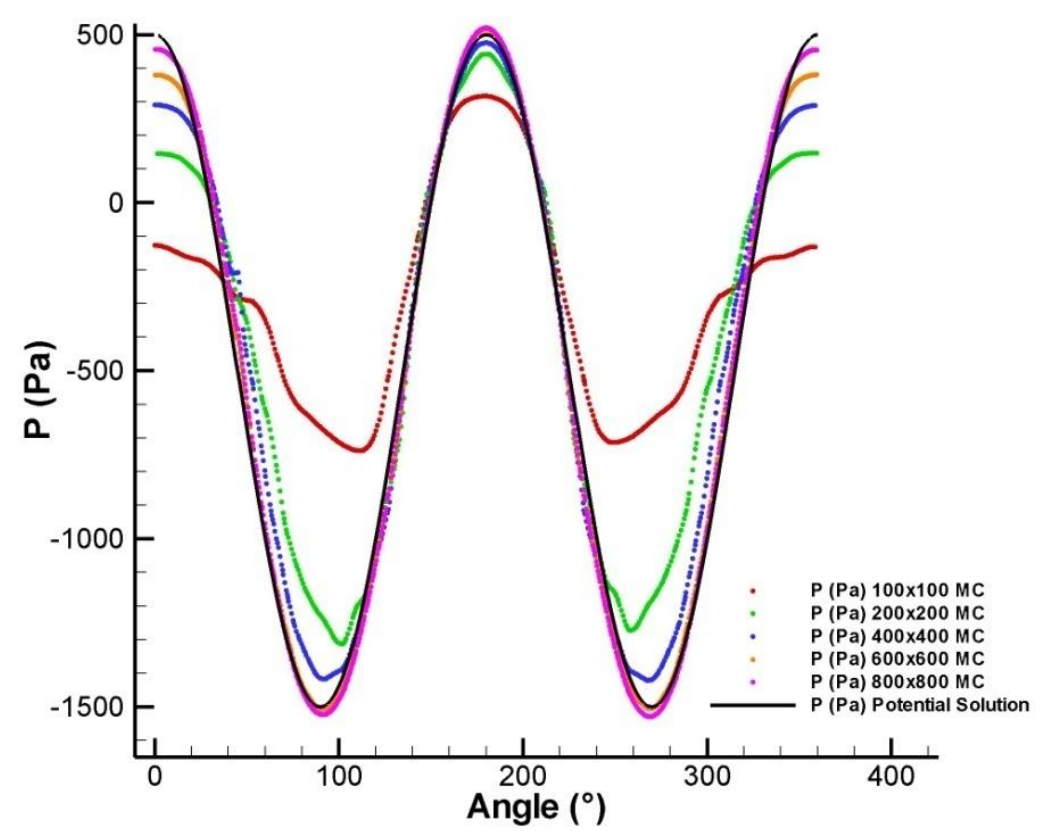

Figure 2. Local steady pressure around the cylinder.

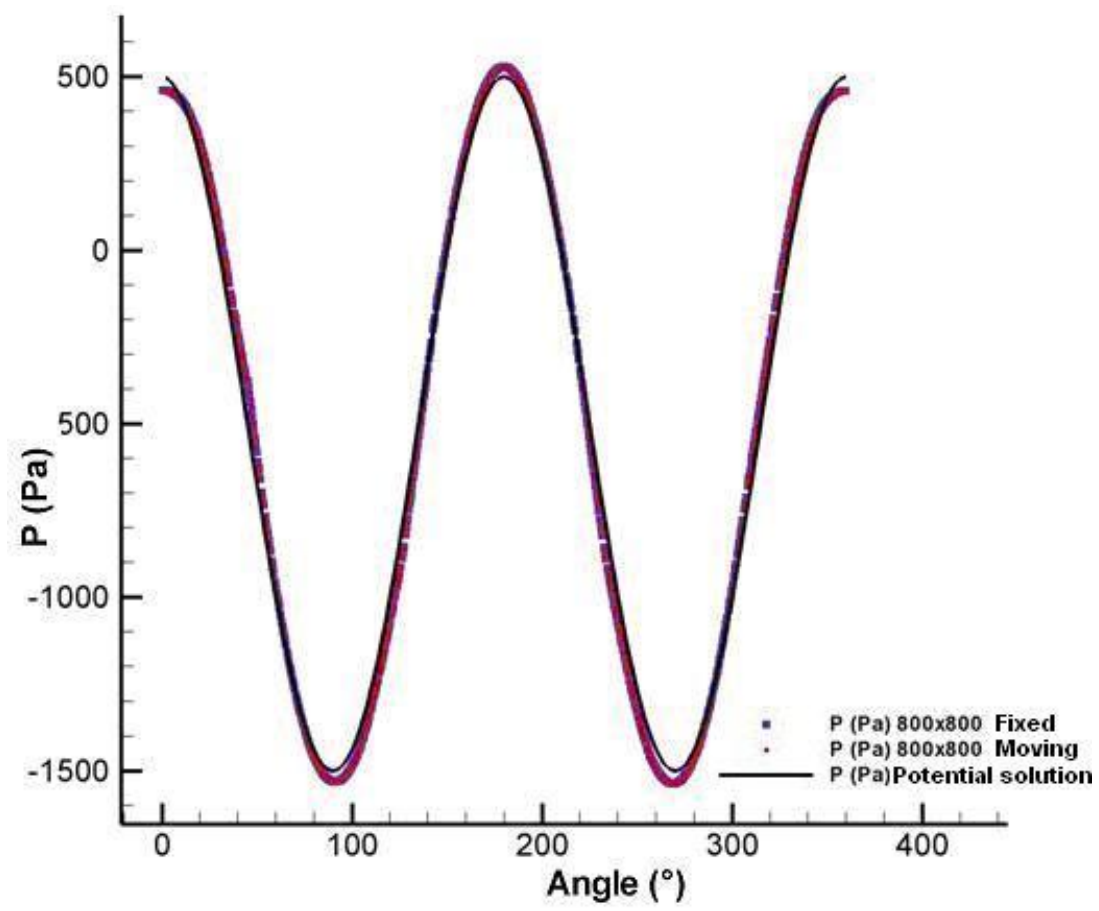

Figure 3. Local pressure comparison between fixed and moving cylinder

Figure 3 illustrates that a fully validated 2-D $2^{\text {nd }}$ order scheme is obtained with this cut-cell based wall boundary condition algorithm. 


\section{TWO-FLUID CARTESIAN-GRID FINITE-VOLUME CHARACTERISTIC FLUX MODEL}

Linearizing perfect fluid equation to obtain compressible jump condition for two fluids interface:

$$
\left\{\begin{array}{l}
\rho \frac{\partial u}{\partial t}+\frac{\partial p}{\partial x}=0 \\
\frac{\partial p}{\partial t}+\rho c^{2} \frac{\partial u}{\partial x}=0
\end{array} \Leftrightarrow\left(\begin{array}{l}
u \\
p
\end{array}\right)_{t}+\left(\begin{array}{cc}
0 & \frac{1}{\rho} \\
\rho c^{2} & 0
\end{array}\right) \cdot\left(\begin{array}{l}
u \\
p
\end{array}\right)_{x}=0\right.
$$

After time derivation:

$$
\left(\begin{array}{l}
u \\
p
\end{array}\right)_{t t}=\left(\begin{array}{cc}
0 & \frac{1}{\rho} \\
\rho c^{2} & 0
\end{array}\right)^{2} \cdot\left(\begin{array}{l}
u \\
p
\end{array}\right)_{x x}=\left(\begin{array}{cc}
c^{2} & 0 \\
0 & c^{2}
\end{array}\right) \cdot\left(\begin{array}{l}
u \\
p
\end{array}\right)_{x x}
$$

Pressure and velocity zero order jump condition become:

$$
\begin{array}{ccc}
{[p]=0} & \text { et } & {[u]=0} \\
{[f]=\lim _{x \rightarrow \alpha^{+}} f(x, t)-\lim _{x \rightarrow \alpha^{-}} f(x, t) .}
\end{array}
$$

first order jump condition becomes :

$$
\left[\frac{1}{\rho} \frac{\partial p}{\partial x}\right]=0 \quad \text { et } \quad\left[\rho c^{2} \frac{\partial u}{\partial x}\right]=0
$$

second order jump condition becomes :

$$
\left[c^{2} \frac{\partial^{2} p}{\partial x^{2}}\right]=0 \quad \text { et } \quad\left[c^{2} \frac{\partial^{2} u}{\partial x^{2}}\right]=0
$$

We use here the first order scheme, using zero order compressible jump condition. Our hyperbolic system respects Riemann invariant $\pi^{ \pm}=p \pm \rho c u$. By advecting these terms with a celerity $\pm c$, we obtain:

$$
\left\{\begin{array}{l}
\frac{\partial \pi^{+}}{\partial t}+c \frac{\partial \pi^{+}}{\partial x}=0 \\
\frac{\partial \pi^{-}}{\partial t}-c \frac{\partial \pi^{-}}{\partial x}=0
\end{array}\right.
$$

After linearization:

$$
\left\{\begin{array} { l } 
{ \pi _ { \mathrm { int } } ^ { + } = \pi _ { r } ^ { + } } \\
{ \pi _ { \mathrm { int } } ^ { - } = \pi _ { l } ^ { + } }
\end{array} \Leftrightarrow \left\{\begin{array}{l}
p_{\mathrm{int}}+\rho_{\mathrm{int}} c_{\mathrm{int}} u_{\mathrm{int}}=p_{l}+\rho_{l} c_{l} u_{l} \\
p_{\mathrm{int}}+\rho_{\mathrm{int}} c_{\mathrm{int}} u_{\mathrm{int}}=p_{r}+\rho_{r} c_{r} u_{r}
\end{array}\right.\right.
$$

The central wave in this study is necessarily a shock discontinuity leading to the following results: 


$$
\left\{\begin{array}{l}
p *_{R}=p *_{L}=p *^{(1)}=v *_{L}^{(1)}=v *^{(1)} \\
v *_{R}^{(1)}=v{ }^{2}
\end{array}\right.
$$

Following pressure out the left or right characteristic wave, we will have shock or rarefaction waves, with continuity of transversal velocity:

$$
\left\{\begin{array}{l}
v *_{L}^{(2)}=v_{L}^{(2)} \\
v *_{R}^{(2)}=v_{R}^{(2)}
\end{array}\right.
$$

So,

$$
\left\{\begin{array} { l } 
{ p _ { \mathrm { int } } + \rho _ { l } c _ { l } u _ { \mathrm { int } } = p _ { l } + \rho _ { l } c _ { l } u _ { l } } \\
{ p _ { \mathrm { int } } - \rho _ { r } c _ { r } u _ { \mathrm { int } } = p _ { r } - \rho _ { r } c _ { r } u _ { r } }
\end{array} \Leftrightarrow \left\{\begin{array}{l}
p_{\mathrm{int}}=\frac{\rho_{l} c_{l} u_{r}+\rho_{r} c_{r} u_{l}}{\rho_{l} c_{l}+\rho_{r} c_{r}}+\rho_{l} c_{l} \rho_{r} c_{r} \frac{u_{l}-u_{r}}{\rho_{l} c_{l}+\rho_{r} c_{r}} \\
u_{\mathrm{int}}=\frac{\rho_{l} c_{l} u_{l}+\rho_{r} c_{r} u_{r}}{\rho_{l} c_{l}+\rho_{r} c_{r}}+\frac{p_{l}-p_{r}}{\rho_{l} c_{l}+\rho_{r} c_{r}}
\end{array}\right.\right.
$$

\section{VALIDATIONS}

\subsection{2-D biphasic hydrostatic case}

We consider here the two-dimensional case and we seek a solution to the following system:

$$
\left\{\begin{array}{l}
\frac{\partial P}{\partial y}-g \rho(y)=0 \\
P-P_{0}=\frac{\rho_{0} c_{0}^{2}}{\gamma}\left[\left(\frac{\rho}{\rho_{0}}\right)^{\gamma}-1\right]
\end{array}\right.
$$

which defines a new initial problem:

$$
\left\{\begin{array}{l}
\rho(y)=\rho_{0}\left(1-\frac{g(\gamma-1) y}{c_{0}^{2}}\right)^{\frac{1}{\gamma-1}} \\
P(y)=P_{0}+\frac{\rho_{0} c_{0}^{2}}{\gamma}\left[\left(1-\frac{g(\gamma-1) y}{c_{0}^{2}}\right)^{\frac{\gamma}{\gamma-1}}-1\right]
\end{array}\right.
$$




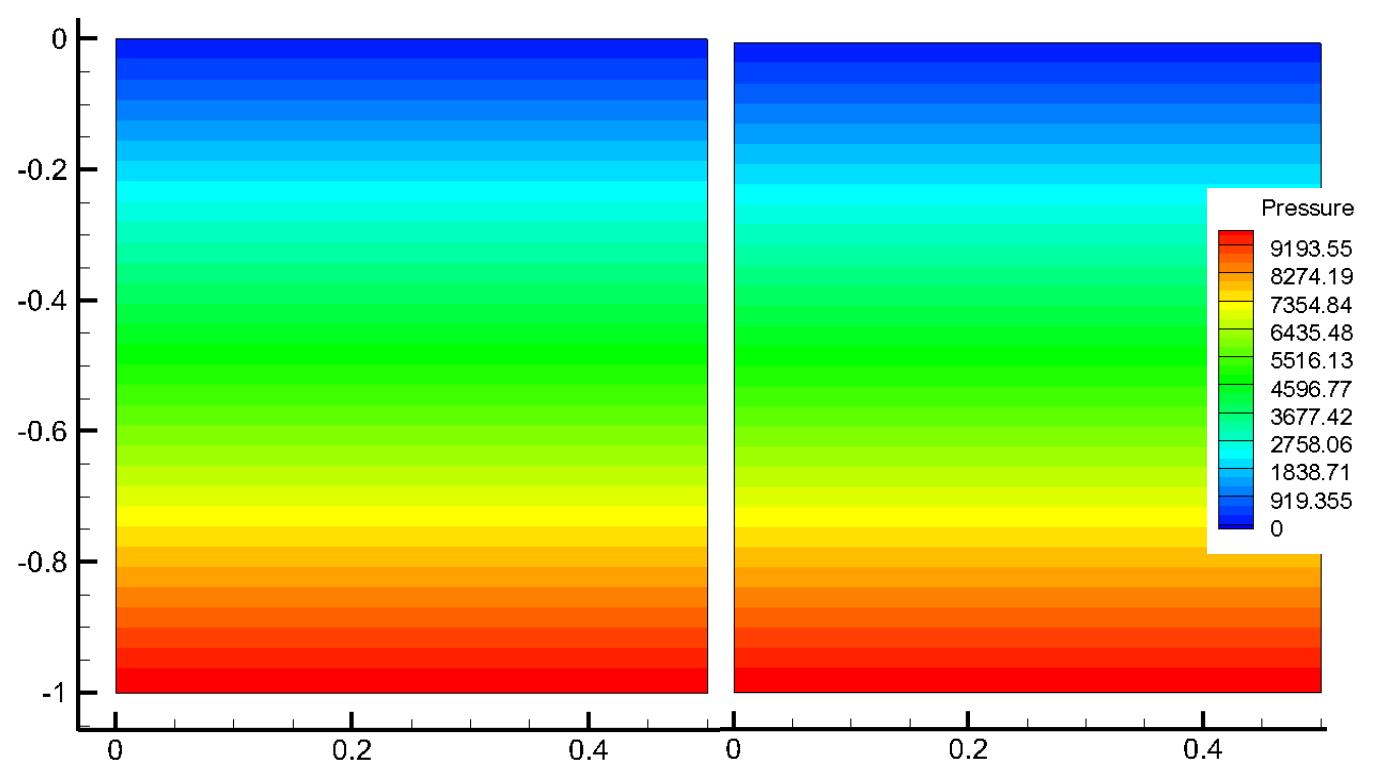

Figure 4. Pressure field at $\mathrm{t}=0 \mathrm{~s}$ (left) and $\mathrm{t}=20 \mathrm{~s}$ (right).
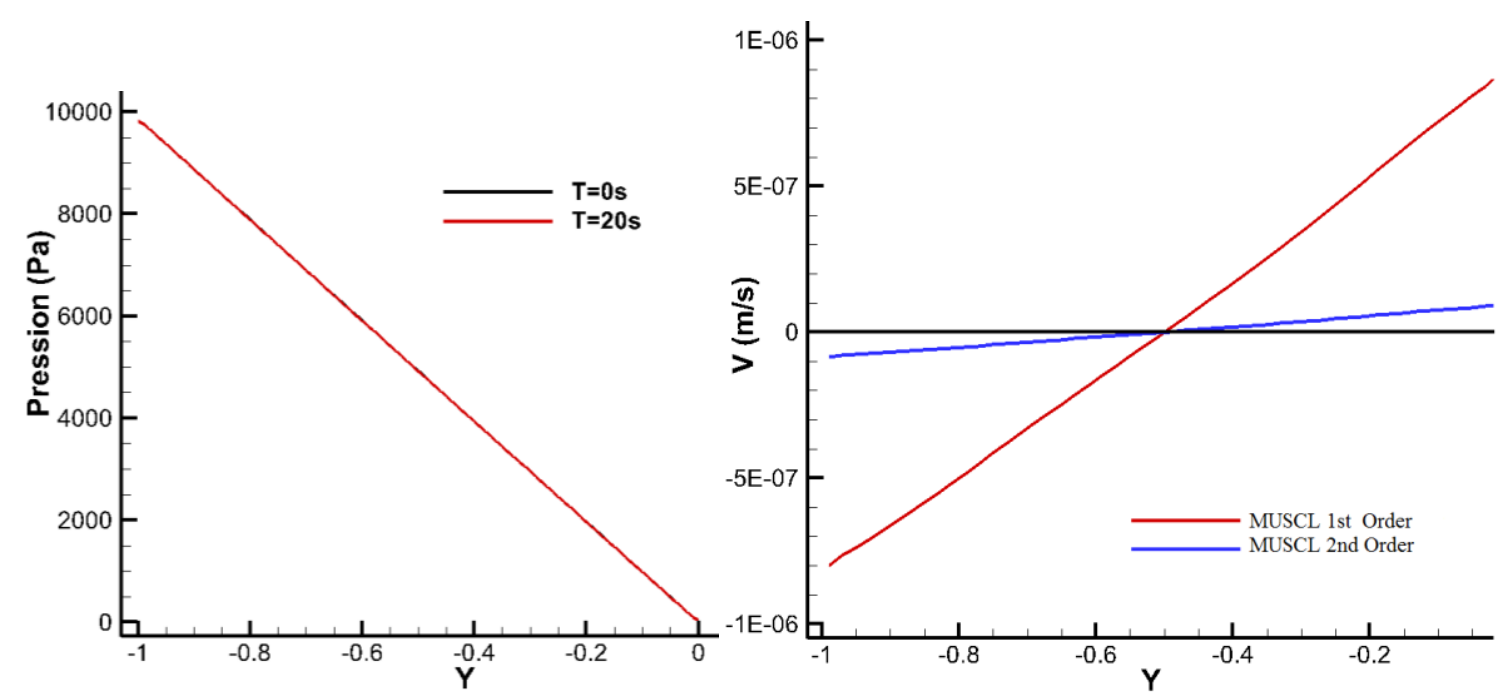

Figure 5. Pressure field comparison between 0s and 20s (left). Velocity field comparison between $1^{\text {st }}$ and $2^{\text {nd }}$ order MUSCL scheme at $\mathrm{t}=20$ s (right).

A good agreement is observed between the analytical and the numerical solution. The presence of a low velocity gradient is explained by the error of the MUSCL extrapolation of the pressure on the walls of our tank. Indeed the whole field is initialized at the cell center. The treatment of boundary conditions, requiring a calculation projected on the border of approximation error, induces a very weak stationarity in vertical velocity. Nevertheless the pressure oscillations measured are negligible (Figure 4-5).

\subsection{2-D low-Mach biphasic linear slohing}

We now consider a two-dimensional test case to assess the correct behavior in low-Mach regime in the case of a linear biphasic sloshing tank, which solution can be compared with a generate potential solution. This sloshing tank is a rectangular domain, filled with water and 
air under the action of both the gravity $\vec{g}$ and a transverse acceleration of modulus $g / 100$, as follows:

$$
\vec{S}=\mid \begin{aligned}
& -g / 100 \\
& -g
\end{aligned}
$$

Water and air are initialized with their nominal physical properties, the dimensions of the sloshing tank are specified in figure 6 below, with $\mathrm{H}_{\text {water }} / \mathrm{L}=1$ and $\mathrm{H}_{\text {tank }} / \mathrm{L}=2.25$. The elevation of the free surface can be evaluated analytically by potential theory as follows [1718]:

$$
\left\{\begin{array}{l}
\xi(x, t)=\frac{a_{0}}{g}\left(x-\frac{L}{2}+\sum_{n \geq 0} \frac{4}{L k_{2 n+1}^{2}} \cos \left(\omega_{2 n+1} t\right) \cos \left(k_{2 n+1} x\right)\right) \\
k_{n}=\frac{n \pi}{L}, \ldots \ldots \ldots . ., \omega_{n}^{2}=\frac{g k_{n}\left(\rho_{2}-\rho_{1}\right)}{\rho_{1} \operatorname{coth}\left[k_{n}\left(H_{\tan k}-H_{\text {water }}\right)\right]+\rho_{2} \operatorname{coth}\left[k_{n} H_{\text {water }}\right]}
\end{array}\right.
$$

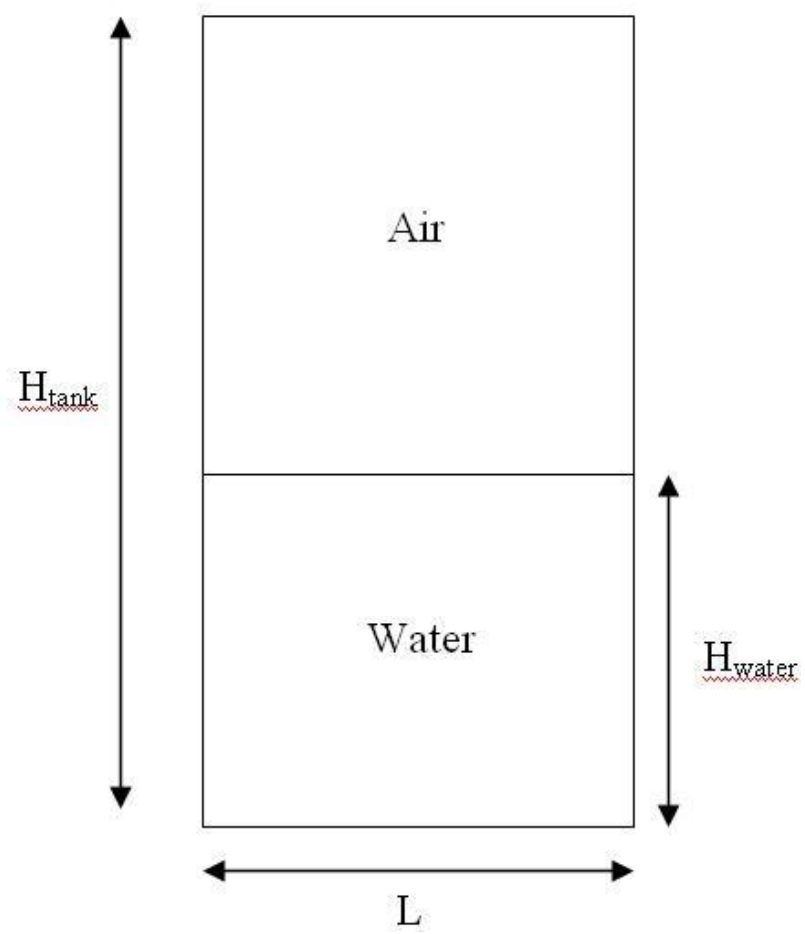

Figure 6. Initial configuration

A convergence study was carried out in grids: three discretizations applied on uniform grids were chosen: 40x20, 90x45 and 180x80 cells. The displacement of the interface (about $1 \mathrm{~cm}$ on each side) is completely included in the two cells for finer grid while the interface moves in a half cell of the coarser grid.

The results are presented (Figure 7), when the rise of the free surface on both sides is compared with the analytical potential solution. The results show a good agreement on fine grids and a significant dissipation on coarse ones, nevertheless it should be emphasized that a low Mach number (around $6.10^{-4}$ ) is observed here, explaining the results obtained. These 
results can be compared with those obtained with a Godunov type solver. One way around this limitation is to artificially increase the Mach number by decreasing the speed of sound in the EOS, here taken as $30 \mathrm{~m} / \mathrm{s}$.
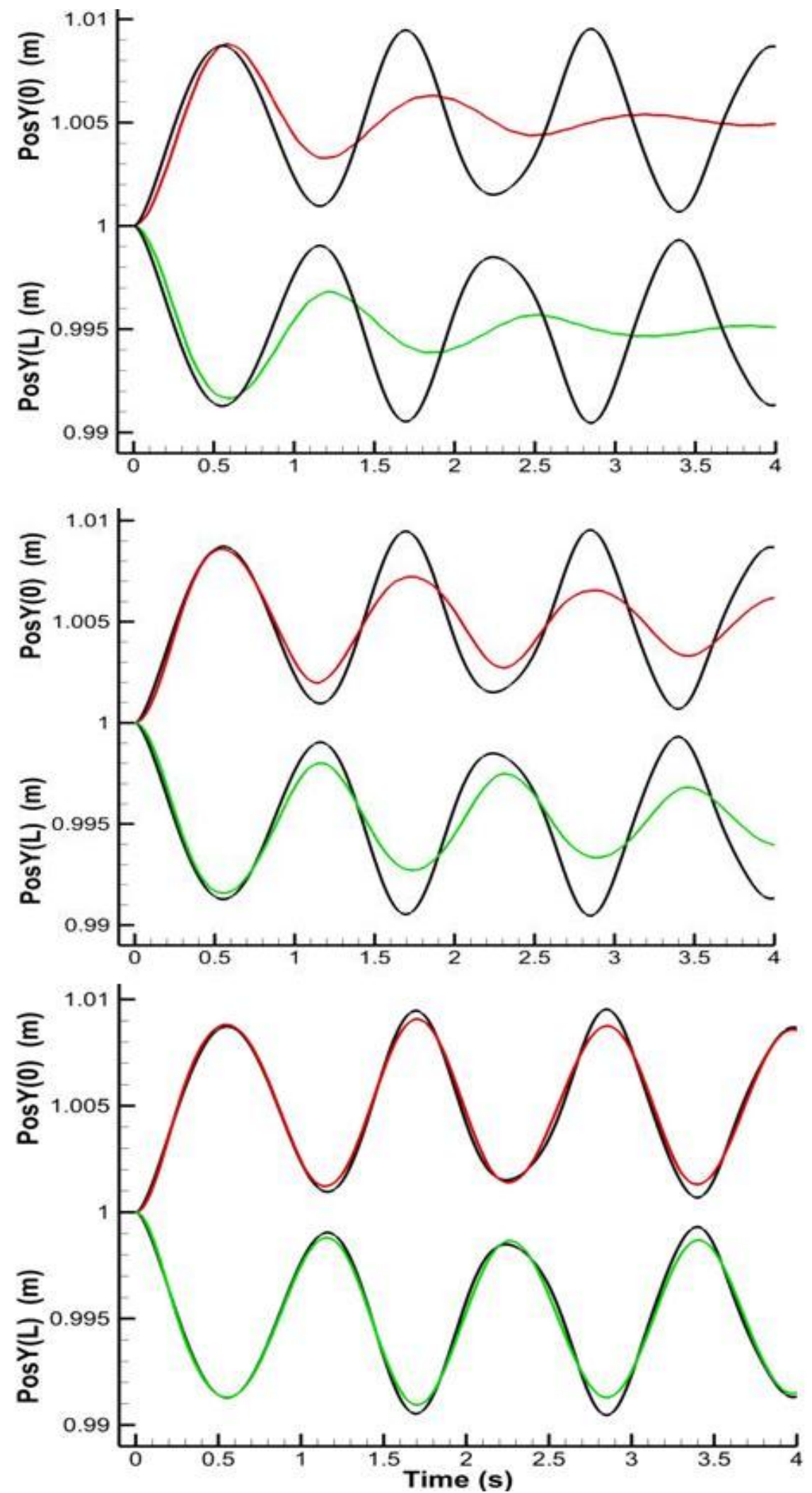

Figure 7. Linear slohing Ma= 10-5 with 2 nd order FVCC on uniform grid from top 40*20, $90 * 45,180 * 80$. 


\section{CONCLUSION}

A new solver based on an explicit Finite Volume solution of hydrodynamic equations is developed. It is based on an explicit solution relying on a fixed non-conform Cartesian grid into which bodies can freely move thanks to a dedicated cut-cell technique. First validations are presented showing encouraging results in the case of a body freely moving in the fixed grid. The solver is now being extended to handle interfaces by means of a fully conservative technique. First tests prove the effectiveness of the conservative technique developed. It will now be compared to other method results on representative marine applications. Further developments will deal with automatic refinement of the mesh which will be eased by the Cartesian nature of the grid.

\section{REFERENCES}

[1] J.-M. Ghidaglia, A. Kumbaro, G. Le Coq, Une méthode volumes finis à flux caractéristiques pour la résolution numérique des systèmes hyperboliques de lois de conservation, C.R. Acad. Sc. Paris, Vol.322, p. 981-988, (1996).

[2] C. Leroy and al., Development of a Cartesian-Grid Finite-Volume Characteristic Flux Model for Marine Applications", Materials Science and Engineering, Vol 10, (2010).

[3] B.V Leer, Towards the ult,mate conservative difference scheme V. A second order sequel to Godunov's methods, J. Comput Phys. 39, 101-136, (1979)

[4] J. P. Vila, "On particle weighted methods and SPH," Mathematical Models and Methods in Applied Sciences, vol. 9, pp. 161-210, 1999.

[5] Le Touzé D., Oger G. \& Alessandrini B., "Smoothed Particle Hydrodynamics simulation of fast ship flows", Proc. of $27^{\text {th }}$ Symp. on Naval Hydrodynamics (SNH 2008), Seoul, Korea, 2008.

[6] A. Kurganov, E Tadmor. Solution of Two-Dimensional Riemann Problems for Gas Dynamics without Riemann Problem Solvers.

[7] M. Berger, R. J. Leveque. Stable boundary conditions for Cartesian grid calculations. Computing Systems in Engineering, 1:305-311, 1990.

[8] D.M. Ingram, D.M. Causon, C.G. Mingham, Developments in Cartesian cut cell methods, Mathematics and Computers in Simulation 61 (2003) 561-572

[9] J. Amanatides, A. Woo. A Fast Voxel Traversal Algorithm for Ray Tracing Dept. of Computer Science University of Toronto, Ontario, Canada.

[10] B.V Leer, Towards the ultimate conservative difference scheme V. A second order sequel to Godunov's methods, JCP, (1979)

[11]D.M. Ingram, D.M. Causon, Developments in Cartesian cut cell methods, Mathematics and Computers in Simulation 61 (2003) 561-572

[12] J. Amanatides, A. Woo. A Fast Voxel Traversal Algorithm for Ray Tracing Dept. of Computer Science University of Toronto, Canada.

[13] Toro, Eleuterio F. (1999). Riemann Solvers and Numerical Methods for Fluid Dynamics. Berlin: Springer Verlag.

[14] A. Kurganov, E Tadmor. Solution of Two-Dimensional Riemann Problems for Gas Dynamics without Riemann Problem Solvers. 
[15] CW Shu, S. Osher, Efficient implementation of essentially non-oscillatory shock-capturing schemes, JCP, 77 (1988), pp. 439-471

[16] Coirier, W. J. and Powell, K. G.: An Accuracy Assessment of Cartesian-Mesh Approaches for The Euler Equations, J. Comput. Physics, 117 (1995), pp. 121-131

[17] G. Chanteperdrix, Modélisation et simulation numérique d'écoulements diphasiques à interface libre. Application à l'étude des mouvements de liquides dans les réservoirs de véhicules spatiaux., PhD Thesis, Ecole Nationale Supérieure de l'Aéronautique et de l'Espace, 2004.

[18] G. Chanteperdrix, J.-L. Estivalezes, Test-case number 34: Two-dimensional sloshing in cavity - an exact solution, 2003. Http://test.interface.free.fr/Case34.pdf. 